\title{
Effects of prazosin on fertility of male rats
}

\author{
W. D. Ratnasooriya and R. M. Wadsworth* \\ Department of Zoology, University of Colombo, Colombo 3, Sri Lanka and ${ }^{*}$ Department of \\ Physiology and Pharmacology, University of Strathclyde, Glasgow G1 IXW, U.K.
}

\begin{abstract}
Summary. Silastic rods containing $25 \%$ or $50 \%$ prazosin $\mathrm{HCl}$ were inserted adjacent to the epididymis of rats. Silastic collars containing $25 \%$ prazosin $\mathrm{HCl}$ were placed around the vas deferens of rats. Treated males were infertile or subfertile for $6-9$ weeks after insertion of these rods or collars. The number of spermatozoa observed in the vaginal smears was reduced. It is concluded that the antifertility effect of prazosin results mainly from inhibition of ejaculation.
\end{abstract}

\section{Introduction}

The vas deferens is filled with spermatozoa from the cauda epididymidis during sexual stimulation in the rabbit, and the vasa become the direct source of spermatozoa at the time of emission. During sexual rest there is a redistribution of spermatozoa towards the epididymis and proximal vas deferens, resulting in conservation of spermatozoa (Prins \& Zaneveld, 1979, 1980). The vas deferens and epididymis are densely innervated by adrenergic nerves, but there is also some evidence for a non-adrenergic transmitter in the vas deferens in several species (Sjöstrand, 1981). The $\alpha$-adrenoceptor selective antagonist prazosin has been reported to reduce contractions of the rat vas deferens produced by trains of stimuli applied to the motor nerve (Doggrell, 1981). When the nerve supply to the rat vas deferens is excited with single shocks, biphasic contractions are produced; prazosin antagonizes the second of these phases, but has no effect on the first (McGrath, 1978). However, inhibition by prazosin of contractions produced by trains may indirectly involve the first component in addition to the second (French \& Scott, 1982). The physiological frequency of excitation of the nerves to the vas deferens is unknown, but the results of Doggrell (1981) raise the possibility that prazosin might inhibit contractions of the vas deferens during mating. We have therefore studied the effect of prazosin on fertility using male rats and a method of local administration.

\section{Materials and Methods}

Animals. Healthy adult laboratory bred rats of mixed strain and proven fertility (males weighing 275-325 g and females weighing $200-250 \mathrm{~g}$ ) were used. They were housed in a well ventilated animal house at a temperature of $28-30^{\circ} \mathrm{C}$ with a natural photoperiod (about $12 \mathrm{~h}$ light and $12 \mathrm{~h}$ dark daily). All rats received food (rat pellets and green leaves) and tap water ad libitum.

Construction of sustained-released drug delivery systems. Silastic formulations containing $25 \%$ prazosin $\mathrm{HCl}$ were constructed in the form of rods or collars by mixing known weights of powdered prazosin $\mathrm{HCl}$ and polysiloxane polymer (Silastic 382 Medical Grade Elastomer; Dow Corning Ltd, Midland, MI, U.S.A.) using a pestle and mortar (Ratnasooriya, Wadsworth \& Gilmore, 1979; 
Ratnasooriya, Gilmore \& Wadsworth, 1980). Completed rods were $2 \mathrm{~mm}$ in diameter, $8-10 \mathrm{~mm}$ long and weighed $30-50 \mathrm{mg}$. The collars were $10-12 \mathrm{~mm}$ long, $8 \mathrm{~mm}$ in diameter with a central hole of about $2.8 \mathrm{~mm}$ and weighed about $500 \mathrm{mg}$. A longitudinal incision was made along the wall of the collar to permit insertion around the vas deferens. Drug-free rods and collars consisting only of Silastic were also made.

Insertion of rods adjacent to epididymis and collars around the vas deferens. Insertion of rods or collars was performed using aseptic precautions under mild ether anaesthesia. A rod containing $25 \%$ prazosin $\mathrm{HCl}$ (12 rats), $50 \%$ prazosin $\mathrm{HCl}$ (6 rats) or a drug-free rod (10 rats) was placed adjacent to each epididymis via an incision made in the scrotal sac and in the tunica vaginalis of each side as described by Ratnasooriya et al. (1980). In another series of rats, the prostatic ends of both vasa deferentia were exposed through a $2-3 \mathrm{~cm}$ midline incision in the abdominal musculature (Ratnasooriya et al., 1979). A single collar containing $25 \%$ prazosin $\mathrm{HCl}$ (6 rats) or a drug-free collar (8 rats) was then fitted around each vas deferens which was then returned to its former position. After both of these surgical procedures, the wounds were treated with Polybactrin powder spray (Calmic Ltd, Berkhamstead, Herts, U.K.), the incisions were sutured and the animals were then allowed to recover. The day of insertion of the device was designated as Day 0.

Assessment of fertility. Libido, ejaculatory ability and fertility of the operated animals were tested at Days 3 and 7 and then at weekly intervals by pairing each male overnight with a sexually experienced pro-oestrous female with a regular oestrous cycle as determined by vaginal smears. The sexual behaviour pattern of the paired rats was noted 3-4 h later. Insemination was confirmed by the presence of spermatozoa in the vaginal smear on the following morning $(07: 00-08: 00 \mathrm{~h})$. If spermatozoa were present a rough estimate of their number and motility was made. In the absence of spermatozoa, daily vaginal smears were continued to determine the occurrence of pregnancy or pseudopregnancy. At 8-12 days post coitum, the females were laparotomized and the number of embryos present in each uterine horn was recorded.

Motility of epididymal spermatozoa. The motility of epididymal spermatozoa was assessed in 8 rats, each of which was fitted with one $25 \%$ prazosin $\mathrm{HCl}$ rod adjacent to one epididymis and one drug-free rod adjacent to the contralateral epididymis. At Day 7 these rats were lightly anaesthetized and spermatozoa from a portion of each cauda epididymidis were extruded into $0.9 \%$ $(\mathrm{w} / \mathrm{v}) \mathrm{NaCl}$ solution and their motility was scored using a subjective scale from 0 (immotile) to 5 (greatest motility ever observed).

\section{Results}

The appetite and general health of the rats remained normal throughout the study. In the whole period of the study, two animals died; in neither case was the cause of death known, but there was no apparent relationship to the treatment given. Some drowsiness was apparent for 3-4 $\mathrm{h}$ after the operation in most of the rats fitted with prazosin-containing rods or collars. Drowsiness has also been noted as a side effect with clinical use of prazosin (Brogden, Heel, Speight \& Avery, 1977). Libido was not affected by prazosin since the treated animals remained able to mate and their courting behaviour appeared normal.

Prazosin reduced fertility when applied in the form of collars (Table 1) or rods (Table 2). Out of 117 control matings, all resulted in pregnancy, and in only 2 cases were there fewer than 5 embryos/rat. In contrast, every treated animal was subfertile (4 embryos or less) on at least one occasion. The data were summarized by calculating at each time interval the fertility index, i.e. total number of embryos/number of matings. These results are given in Table 3, which shows that the fertility index was reduced by prazosin. The high-dose rods had a greater effect than did the low-dose rods. Statistical analysis of these results was made using the Mann-Whitney test (Siegel, 
Table 1. Effect on fertility (no. of embryos from each pairing) in male rats of prazosin-containing Silastic collars

\begin{tabular}{|c|c|c|c|c|c|c|c|c|c|c|c|c|}
\hline & \multicolumn{12}{|c|}{ Time after operation } \\
\hline & \multirow{2}{*}{$\frac{\text { Days }}{3}$} & \multicolumn{11}{|c|}{ Weeks } \\
\hline & & 1 & 2 & 3 & 4 & 5 & 6 & 7 & 8 & 9 & 10 & 11 \\
\hline Control collars & $\begin{array}{c}6 \\
6 \\
8 \\
8 \\
8 \\
8 \\
6 \\
\text { NM }\end{array}$ & $\begin{array}{l}6 \\
8 \\
8 \\
8 \\
6 \\
6 \\
8 \\
6\end{array}$ & $\begin{array}{l}2 \\
8 \\
6 \\
8 \\
\mathrm{NM} \\
7 \\
6 \\
7\end{array}$ & $\begin{array}{c}8 \\
3 \\
7 \\
8 \\
\mathrm{NM} \\
8 \\
\mathrm{NM} \\
6\end{array}$ & $\begin{array}{c}70 \\
7 \\
8 \\
5 \\
8 \\
8 \\
6 \\
10\end{array}$ & $\begin{array}{l}-\overline{8} \\
-6 \\
8 \\
8 \\
7 \\
9\end{array}$ & $\begin{array}{c}6 \\
\frac{6}{8} \\
8 \\
7 \\
10 \\
9 \\
9\end{array}$ & $\begin{array}{c}6 \\
8 \\
\text { NM } \\
7 \\
- \\
- \\
- \\
-\end{array}$ & $\begin{array}{l}6 \\
8 \\
8 \\
7 \\
- \\
- \\
-\end{array}$ & $\begin{array}{l}7 \\
7 \\
8 \\
8 \\
- \\
- \\
-\end{array}$ & $\begin{array}{c}7 \\
8 \\
7 \\
\mathrm{NM} \\
- \\
- \\
-\end{array}$ & $\begin{array}{c}8 \\
10 \\
8 \\
10 \\
- \\
- \\
- \\
-\end{array}$ \\
\hline Prazosin $25 \%$ & $\begin{array}{c}\text { NM } \\
\text { NM } \\
0 \\
70 \\
5 \\
\text { NM }\end{array}$ & $\begin{array}{l}0 \\
7 \diamond \\
7 \\
0 \\
0 \\
\mathrm{NM}\end{array}$ & $\begin{array}{c}\text { NM } \\
0 \\
2 \\
5 \\
\text { NM } \\
\text { NM }\end{array}$ & $\begin{array}{l}2 \diamond \\
0 \\
5 \diamond \\
0 \\
8 \diamond \\
0\end{array}$ & $\begin{array}{l}0 \diamond \\
9 \\
8 \\
0 \\
8 \\
-\end{array}$ & $\begin{array}{l}6 \\
8 \\
8 \\
60 \\
8 \\
0\end{array}$ & $\begin{array}{l}5 \\
6 \\
6 \\
8 \\
6 \\
0\end{array}$ & $\begin{array}{c}9 \\
7 \\
\mathrm{NM} \\
0 \\
8 \\
0 \diamond\end{array}$ & $\begin{array}{c}9 \\
\frac{6}{7} \\
\frac{7}{0} \\
0\end{array}$ & $\begin{array}{l}9 \\
5 \\
\frac{8}{8} \\
8 \\
5\end{array}$ & $\begin{array}{c}- \\
\overline{-} \\
7 \\
\mathrm{NM} \\
4\end{array}$ & $\begin{array}{l}- \\
- \\
- \\
8 \\
5\end{array}$ \\
\hline
\end{tabular}

* This male died on Day 52.

$\mathrm{NM}=$ mating did not take place at this pairing; when treated animals were not paired this is indicated by - .

$\checkmark$, approximately normal number of spermatozoa in the vaginal smear; $\diamond$, approximately half normal number of spermatozoa in vaginal smear: $\diamond$, only a few spermatozoa in vaginal smear; no symbol = no spermatozoa in vaginal smear.

1956) and comparing each treatment group with the appropriate control group at each time interval. Over the first 5 or 6 weeks after operation, the fertility index was significantly reduced in most treatment groups, although in some of the rats fitted with collars the number of matings was too small for the effect to be significant statistically (Table 3 ).

In the fertility studies, the numbers of spermatozoa in the vaginal smear were counted approximately (Tables $1 \& 2$ ). For each mating these results were analysed by classifying the number of spermatozoa as normal or reduced, and comparisons were made between control and treated groups at each time interval (Fisher Exact Test, $P<0.05$ taken as significant). Rods of $25 \%$ prazosin $\mathrm{HCl}$ significantly increased the proportion of rats with hypozoospermia at 3 days. In animals fitted with $50 \%$ prazosin $\mathrm{HCl}$ rods or with $25 \%$ prazosin $\mathrm{HCl}$ collars, the number of rats with hypozoospermia was significantly different from control at 3 days and at 1,2 and 3 weeks. When present, ejaculated spermatozoa were motile, although a proportion of them was decapitated in most experiments (the collars caused more marked decapitation than did the rods).

In a separate group of experiments, the motility of epididymal spermatozoa was assessed in 8 rats. At 1 week after operation, motility on the control side was $4.9 \pm 0.13$ and on the side treated with a $25 \%$ prazosin $\mathrm{HCl}$ rod motility was $4.25 \pm 0.25(P>0.05$, Mann-Whitney test $)$.

\section{Discussion}

Prazosin reduced fertility whether applied to the vas deferens or epididymis. There was no effect on health or appetite. We have no conclusive evidence about the mechanism of this infertility effect, but it is possible to exclude certain potential sites of action. Prazosin treatment did not affect mating or courting behaviour and is unlikely therefore to have reduced plasma testosterone concentrations. At least in the first few weeks, the effect could not have been due to inhibition of spermatogenesis. Although some spermatozoa were decapitated, this was not sufficient to account 
Table 2. Effect on fertility (no. of embryos from each pairing) in male rats of prazosin-containing Silastic rods

\begin{tabular}{|c|c|c|c|c|c|c|c|}
\hline & & & Time & er ope & & & \\
\hline & Days & & & & & & \\
\hline & 3 & 1 & $2 \dagger$ & 3 & 4 & 5 & 6 \\
\hline Control rods & $\begin{array}{r}8 \\
7 \\
8 \\
8 \\
9 \\
8 \\
10 \\
8 \\
8 \\
9\end{array}$ & $\begin{array}{r}8 \\
8 \\
7 \\
10 \\
8 \\
8 \\
10 \\
10 \\
8 \\
10\end{array}$ & $\begin{array}{r}8 \\
\text { NM } \\
7 \\
9 \\
8 \\
8 \\
9 \\
10 \\
8 \\
10\end{array}$ & $\begin{array}{c}8 \\
\text { NM } \\
6 \\
- \\
- \\
- \\
11 \\
8 \\
9 \\
8\end{array}$ & $\begin{array}{l}- \\
- \\
- \\
- \\
- \\
5 \bullet \\
8 \bullet \\
9 \bullet \\
6\end{array}$ & $\begin{array}{c}6 \\
6 \\
7 \\
- \\
- \\
- \\
10 \\
8 \\
9 \\
8\end{array}$ & $\begin{array}{l}- \\
- \\
- \\
- \\
- \\
8 \\
9 \\
5 \\
9\end{array}$ \\
\hline Prazosin $25 \%$ rods & $\begin{array}{c}0 \\
0 \\
0 \\
6 \bullet \\
N M \\
5 \\
0 \\
7 \diamond \\
8 \\
0 \diamond \\
5 \\
50\end{array}$ & 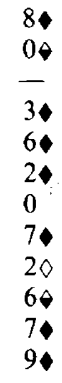 & $\begin{array}{l}6 \bullet \\
0 \diamond \\
5 \diamond \\
6 \bullet \\
0 \\
9 \\
0 \\
5 \\
0 \diamond \\
0 \diamond \\
6 \bullet \\
1 \diamond\end{array}$ & $\begin{array}{l}8 \\
4 \\
1 \\
8 \\
5 \\
5 \\
0 \\
0 \\
8 \\
6 \bullet \\
8 \\
0\end{array}$ & $\begin{array}{c}0 \\
4 \\
5 \\
5 \bullet \\
0 \\
\text { NM } \\
- \\
- \\
- \\
- \\
- \\
-\end{array}$ & $\begin{array}{c}E^{*} \\
- \\
- \\
- \\
- \\
6 \bullet \\
4 \bullet \\
3 \bullet \\
0 \diamond \\
4 \diamond \\
6\end{array}$ & $\begin{array}{l}- \\
- \\
- \\
- \\
- \\
6 \\
6 \\
5 \\
6 \\
8 \\
-\end{array}$ \\
\hline Prazosin $50 \%$ rods & $\begin{array}{c}7 \\
\text { NM } \\
\text { NM } \\
0 \\
8 \\
0\end{array}$ & $\begin{array}{l}6 \\
0 \\
5 \\
0 \\
0 \diamond \\
0\end{array}$ & $\begin{array}{l}1 \\
0 \\
9 \\
3 \\
6 \\
1\end{array}$ & $\begin{array}{l}50 \\
1 \\
6 \bullet \\
0 \\
0 \\
0\end{array}$ & $\begin{array}{l}\frac{40}{-} \\
\frac{7}{-} \\
-\end{array}$ & $\begin{array}{l}- \\
- \\
- \\
-\end{array}$ & $\begin{array}{l}- \\
- \\
-\end{array}$ \\
\hline
\end{tabular}

* This male died at 5 weeks.

† Vaginal smears not taken for the $50 \%$ rod group in this week.

$\mathrm{NM}=$ mating did not take place at this pairing; when treated animals were not paired this is indicated by -

- approximately normal number of spermatozoa in the vaginal smear; $\diamond$, approximately half normal number of spermatozoa in vaginal smear; $\diamond$, only a few spermatozoa in vaginal smear; no symbol = no spermatozoa in vaginal smear.

for the effect on fertility as many normal spermatozoa were also present. The motility of ejaculated or epididymal spermatozoa was not affected adversely.

The site of the antifertility effect was probably extragonadal, since it was apparent in most cases within 7 days and in some rats within 3 days. In view of the method of administration it is likely that the effect was exerted locally on the vas deferens or epididymis. Spermatozoa were present on some occasions even when no embryos were produced, but in general the number of spermatozoa present in the treated animals was reduced. Although a quantitative estimate was not made, the observation of reduced or absent spermatozoa was very consistent, suggesting that prazosin adversely affected either spermatogenesis or ejaculation. In the first few weeks after insertion of the collars (when the antifertility effect was greatest) an antispermatogenic effect would not be apparent. Therefore, the most likely mechanism for the antifertility effect seems to be impairment of ejaculation, which is consistent with the known effect of prazosin to inhibit contraction of the vas deferens (Doggrell, 1981). In clinical use, sexual dysfunction is not a significant side effect with 
Table 3. Summarized data showing the effect on fertility (fertility index) of prazosin-containing Silastic collars and rods

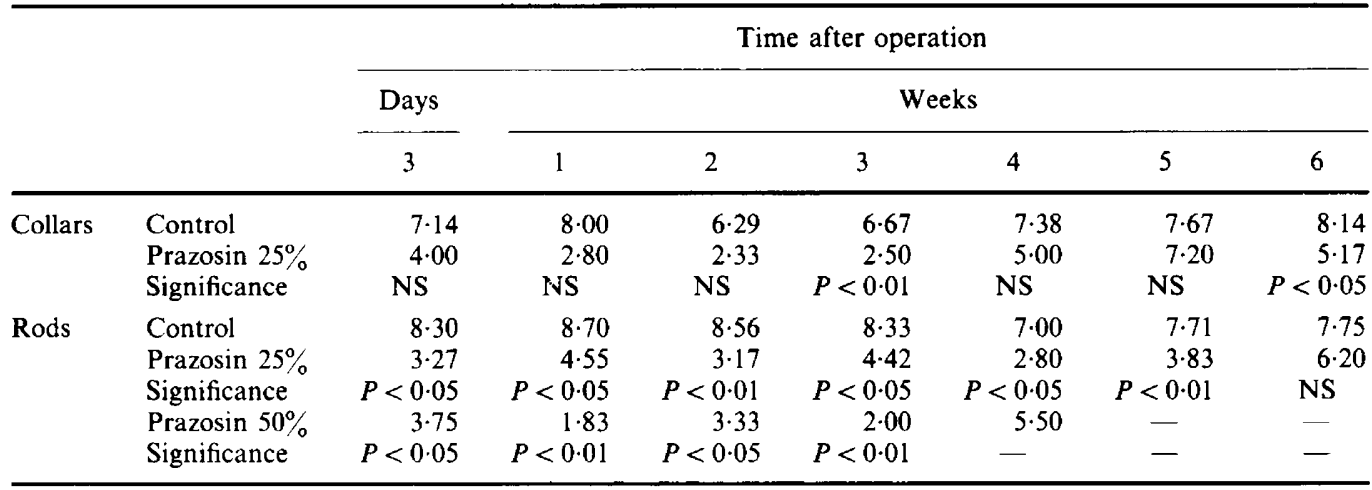

NS, not significant (Mann-Whitney U test).

prazosin (Brogden et al., 1977) possibly because the concentration achieved is below that necessary to inhibit the vas deferens.

From this study we cannot exclude the possibility that all or part of the action of prazosin is caused by alteration of the fertilizing potential or structure (decapitation) of spermatozoa, changes in semen biochemistry, or an effect on the female tract (with transfer via the semen). Comparison of Tables 1 and 2 gives the impression that the collars are having a greater effect on fertility than the $25 \%$ rods, although this could not be demonstrated statistically. If this is a real effect, it is likely to be caused by a difference in the local concentration of prazosin. Since the collars have a greater surface area than the rods, the rate of release of prazosin from them is probably greater. It is not likely to be caused by differences in the innervation of the proximal and distal vas deferens, since in-vitro results show that prazosin has a greater inhibitory effect on the proximal part of the vas deferens in the rat (McGrath, 1978).

It is possible that the decapitation of spermatozoa was caused by inhibition of vas deferens contractions resulting in increased ageing and degeneration of spermatozoa in the epididymis. However, it is doubtful whether the degree of ejaculatory inhibition produced was sufficient to account for this and it is more likely that decapitation is a direct effect of prazosin on spermatozoa.

\section{References}

Brogden, R.N., Heel, R.C., Speight, T.M. \& Avery, G. (1977) Prazosin: a review of its pharmacological properties and therapeutic efficacy in hypertension. Drugs 14, 163-197.

Doggrell, S.A. (1981) Prazosin selectively inhibits the responses to field stimulation in the rat vas deferens. Eur. J. Pharmac. 71, 447-453.

French, A.M. \& Scott, N.C. (1982) Postjunctional $\alpha$ adrenoceptor mediated facilitation of the NANC response in rat vas deferens. Br.J. Pharmac. 77, 476P.

McGrath, J.C. (1978) Adrenergic and 'non-adrenergic' components in the contractile response of the vas deferens to a single indirect stimulus. J. Physiol., Lond. 283, 23-39.

Prins, G.S. \& Zaneveld, L.J.D. (1979) Distribution of spermatozoa in the rabbit vas deferens. Biol. Reprod. 21, $181-185$.

Prins, G.S. \& Zaneveld, L.J.D. (1980) Radiographic study of fluid transport in the rabbit vas deferens during sexual rest and after sexual activity. $J$. Reprod. Fert. 58, 311-319.

Ratnasooriya, W.D., Wadsworth, R.M. \& Gilmore, D.P. (1979) The effect of sympathomimetic drugs on contractility of the vas deferens in vitro and in vivo. $J$. Reprod. Fert. 56, 633-641.

Ratnasooriya, W.D., Gilmore, D.P. \& Wadsworth, R.M. (1980) Effect of local application of sympathomimetic drugs to the epididymis on fertility in rats. $J$. Reprod. Fert. 58, 19-25.

Siegel, S. (1956) Non-parametric Statistics for Behavioral Sciences. McGraw-Hill, New York.

Sjöstrand, N.O. (1981) Smooth muscles of vas deferens and other organs in the male reproductive tract. In Smooth Muscle : an Assessment of Current Knowledge, pp. 367-376. Eds E. Bülbring, A. F. Brading, A. W. Jones \& T. Tomita. Edward Arnold, London. 